American Journal of Immunology 8 (1): 18-26, 2012

ISSN 1553-619X

(C) 2012 Science Publications

\title{
Patterns of Resection among Patients with Hepatic-Only Metastatic Colorectal Cancer a Single Institution Experience and Review
}

\author{
${ }^{1,2}$ K.I. Quintyne, ${ }^{1}$ N. O'Leary, ${ }^{1}$ O. O'Kelly, \\ ${ }^{3}$ D. Waldron, ${ }^{3}$ R. Keane, ${ }^{4}$ J. Geoghegan, ${ }^{5}$ B. Kenny and ${ }^{1,6}$ R.K. Gupta \\ ${ }^{1}$ Department of Medical Oncology, Mid-Western Cancer Centre, \\ Mid-Western Regional Hospital, Limerick, Ireland \\ ${ }^{2}$ Graduate Entry Medical School, University of Limerick, Limerick, Ireland \\ ${ }^{3}$ Department of Surgery, \\ Mid-Western Regional Hospital, Limerick, Ireland \\ ${ }^{4}$ Department of Hepatobiliary Surgery, \\ St. Vincent's University Hospital, Dublin, Ireland \\ ${ }^{5}$ Department of Radiology, Mid-Western Regional Hospital, Limerick, Ireland \\ ${ }^{6}$ Stokes' Institute, University of Limerick, Limerick, Ireland
}

\begin{abstract}
Problem statement: Hepatic-only metastasis in colorectal cancer is not a rare clinical finding and can account for 30\% of cases. However, only 10-25\% of cases are suitable for hepatic resection as part of their treatment pathway. We sought to document our own findings by reviewing patients with hepatic-only disease. Approach: A retrospective analysis was designed to include all patients seen at our institution from 1st January 2000 until 30th June 2010 and information as derived from the patients' records. Results: Forty-four (44) patients were found, with an average age of 60.8 years and a male preponderance. The majority of patients (approximately 57\%) with hepatic-only metastases developed their disease following adjuvant therapy. Better overall survival was seen when a primary tumor had a low grade of histological differentiation and fewer than 3 hepatic lesions appreciated on conventional radiology. Better outcome was seen in patients who underwent hepatic resection. Conclusion: Patients with hepatic-only metastases are not uncommon within our institution. Hepatic resection afforded better outcome and compares favorably with published literature.
\end{abstract}

Key words: Hepatic-only metastatic colorectal cancer, hepatic resection, chemotherapy, hepatic resection, colorectal cancer, male preponderance, majority of patients, retrospective analysis

\section{INTRODUCTION}

Colorectal Cancer (CRC) is a relatively common malignancy and accounts for approximately $12.9 \%$ of all cancer cases diagnosed within the European Union (EU), it is also associated with high mortality and represents almost $12.2 \%$ of all cancer deaths within the EU (Cutsem et al., 2010). Given these findings, the management of this disease and its full clinical spectrum from early stage to advanced stage is well studied and documented.

Less than $20 \%$ of patients with CRC can present with metastases confined to the liver. At least $60 \%$ of patients being managed in the adjuvant setting following definitive colorectal resection may develop hepatic disease; the postulate behind this pattern of spread has been linked to the portal venous drainage from the colon entering the hepatic system (Power and Kemeny, 2010).
Within the last decade there have been significant strides in systemic therapeutic options for advanced disease with the availability of newer agents (including oxaliplatin, irinotecan, bevacizumab and cetuximab) as well as improvements in surgical techniques employed. Combined Modality Treatment (CMT) has led to an improvement in the median overall survival from 8-12 months of 18-24 months (Kopetz et al., 2009).

This is a single institution review of our experience with the management of hepatic-only $\mathrm{CRC}$, who were reviewed for surgical management of their hepatic metastases as part of their CTM; in which we document demographic information including age, sex and stage of presentation, systemic therapy options including chemotherapy and biological therapy, histopathological features, radiological findings, the incidence and outcome of cases involved.

Corresponding Author: K.I. Quintyne, Department of Medical Oncology, Mid-Western Cancer Centre, Mid-Western Regional Hospital, Limerick, Ireland 
Am. J. Immunol., 8 (1): 18-26, 2012

\section{MATERIALS AND METHODS}

A retrospective analysis was designed to include patients referred within the period of 1st January, 2001 to $30^{\text {th }}$ June 2010 to Mid-Western Regional Hospital (MWRH) and data was derived from several sources.

- Mid-Western Cancer Centre (MWCC) Oncology database and electronic patient records

- MWRH cases notes and correspondence

- MWRH pathology reports

- MWRH radiology reports

\section{RESULTS}

There were forty-four (44) patients identified within the designated period, twenty-seven (27) were male and seventeen (17) were female. The mean age at presentation with hepatic metastases was 60.8 years (range of $38-78$ years).

The histopathological features for the cohort were reviewed and all were adenocarcinoma and most were moderately differentiated, with most patients being detected in surveillance following adjuvant therapy and these are displayed in Table 1.

The survival of the cohort based on their histological features was calculated, high histological grade and the presence of metastases at presentation were found to be significant with respect to overall survival, but the nodal status of the primary tumor was not significant, these are displayed in Fig. 1-3.

Statistical review for histological grade at presentation showed that a low grade of differentiation (G1/2 versus G3) had a better overall survival ( $p$ < $0.001)$. The tumor classification stage was also reviewed and the majority of these were $\mathrm{T} 3$ stage $(68.2 \%)$. Statistical review for this variable, showed no difference in overall survival irrespective of the tumour classification stage $(\mathrm{T} 1 / 2$ versus $\mathrm{T} 3)(\mathrm{p}>0.05)$.

Statistical review for nodal status at presentation ( N0/1 versus $\mathrm{N} 2$ ) had no correlation to overall survival $(\mathrm{p}>0.05)$.

The number of hepatic metastases was reviewed for the cohort and the majority of patients had less than 3 lesions at consideration for management and this is displayed in Table 2 and Fig. 4. The number of hepatic metastases was derived from review of a combination of CT, MRI and PET/CT staging scans, for all patients with hepatic-only metastases in the pre-treatment setting.

Statistical review for the number of hepatic metastases showed that patients with less than 3 lesions had a better overall survival ( $\mathrm{p}<0.05$ ).

The therapeutic options for the cohort was reviewed and showed the following preponderances: first line therapy including irinotecan, no involvement of bevacizumab and hepatic lesion resection and these are displayed in Table 3 and Fig. 5-7.

Table 1: Histopathological features for patients with hepatic-only MCRC

\begin{tabular}{lrr}
\hline Histopathological features & Number & Percentage \\
\hline Histology & 44 & $100.00 \%$ \\
Adenocarcinoma & 2 & $4.50 \%$ \\
Histological Grade (G) & 31 & $70.50 \%$ \\
1-well differentiated & 5 & $11.40 \%$ \\
2-moderately differentiated & 6 & $13.60 \%$ \\
3-poorly differentiated & & $2.30 \%$ \\
Not defined & 1 & $15.90 \%$ \\
Tumour Classification (T) & 7 & $68.20 \%$ \\
1-tumour invades submucosa & 30 & $9.10 \%$ \\
2-tumour invades muscularis propria & 4 & $4.50 \%$ \\
3-tumour invades through muscularis propria & 2 & $31.80 \%$ \\
4-tumour directly invades other organ or perforates & & $45.50 \%$ \\
X-primary tumour not assessed & 14 & $20.50 \%$ \\
Nodal Status (N) & 20 & $2.30 \%$ \\
0-no regional lymph node metastases & 9 & \\
1-metastasis in 1 to 3 regional lymph nodes & 1 & $56.80 \%$ \\
2-metastasis in 4 or more regional lymph nodes & & $43.20 \%$ \\
X-regional lymph nodes not assessed & 25 & $13.60 \%$ \\
Metastases at primary presentation (M) & 19 & $11.40 \%$ \\
0 & & $75.00 \%$ \\
1 Epidermal Growth Factor Receptor (EGFR) status & 6 & 5 \\
Wild-type & 33 & \\
Mutant-type & & \\
Not available & &
\end{tabular}


Am. J. Immunol., 8 (1): 18-26, 2012

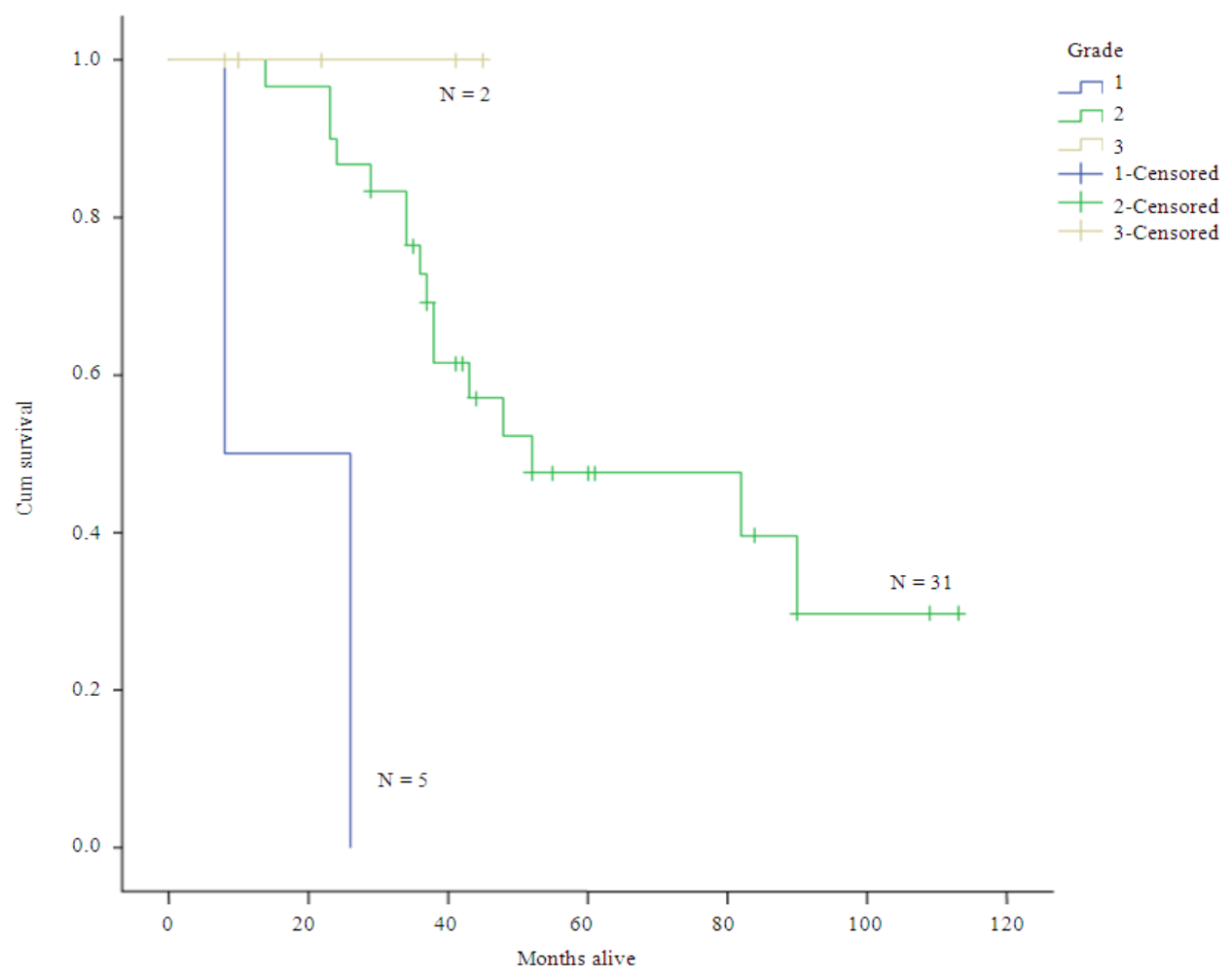

Fig. 1: Kaplan-meier plot showing overall survival with respect to histological grade

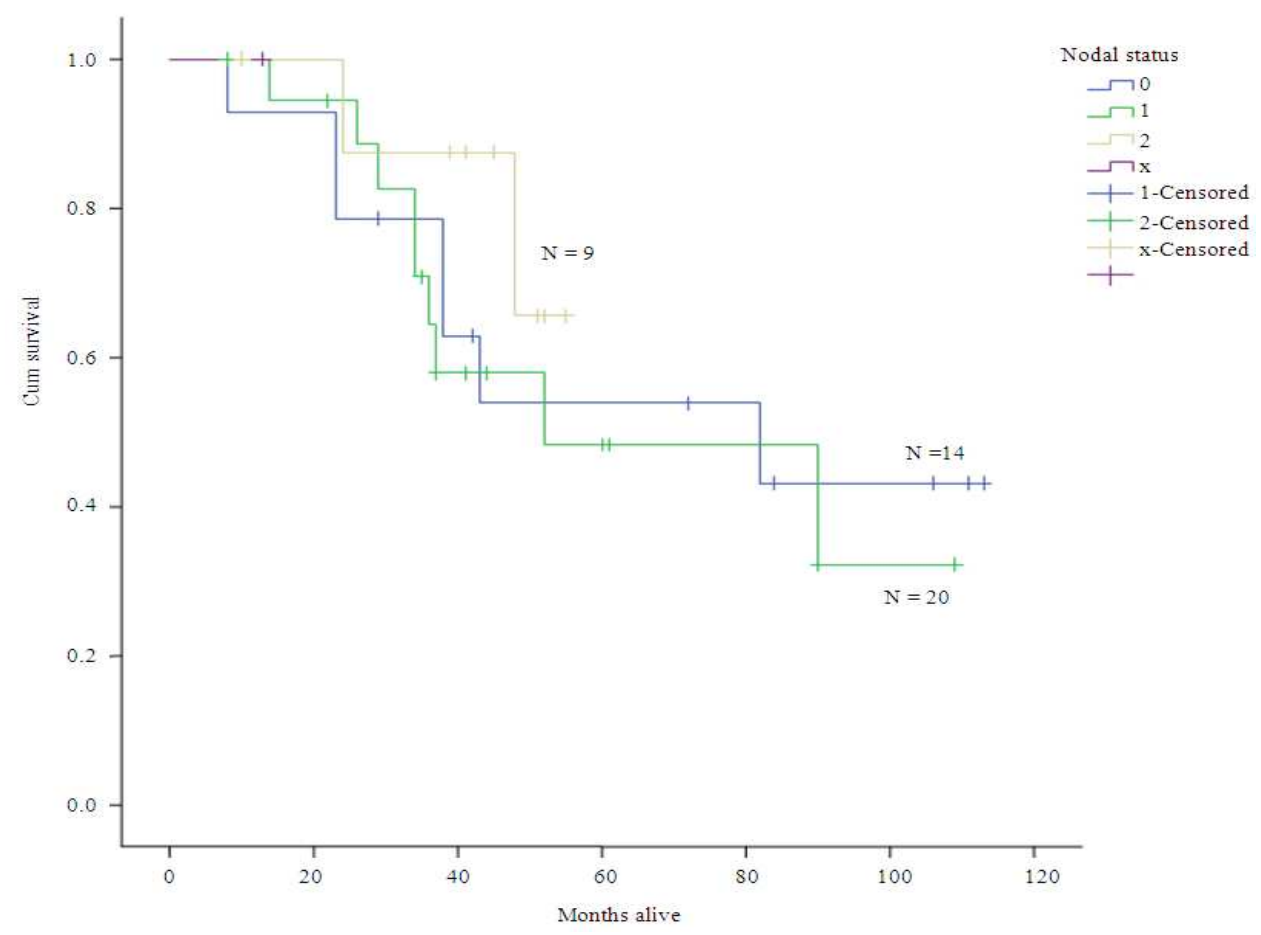

Fig. 2: Kaplan-Meier plot showing overall survival with respect to nodal status 
Am. J. Immunol., 8 (1): 18-26, 2012

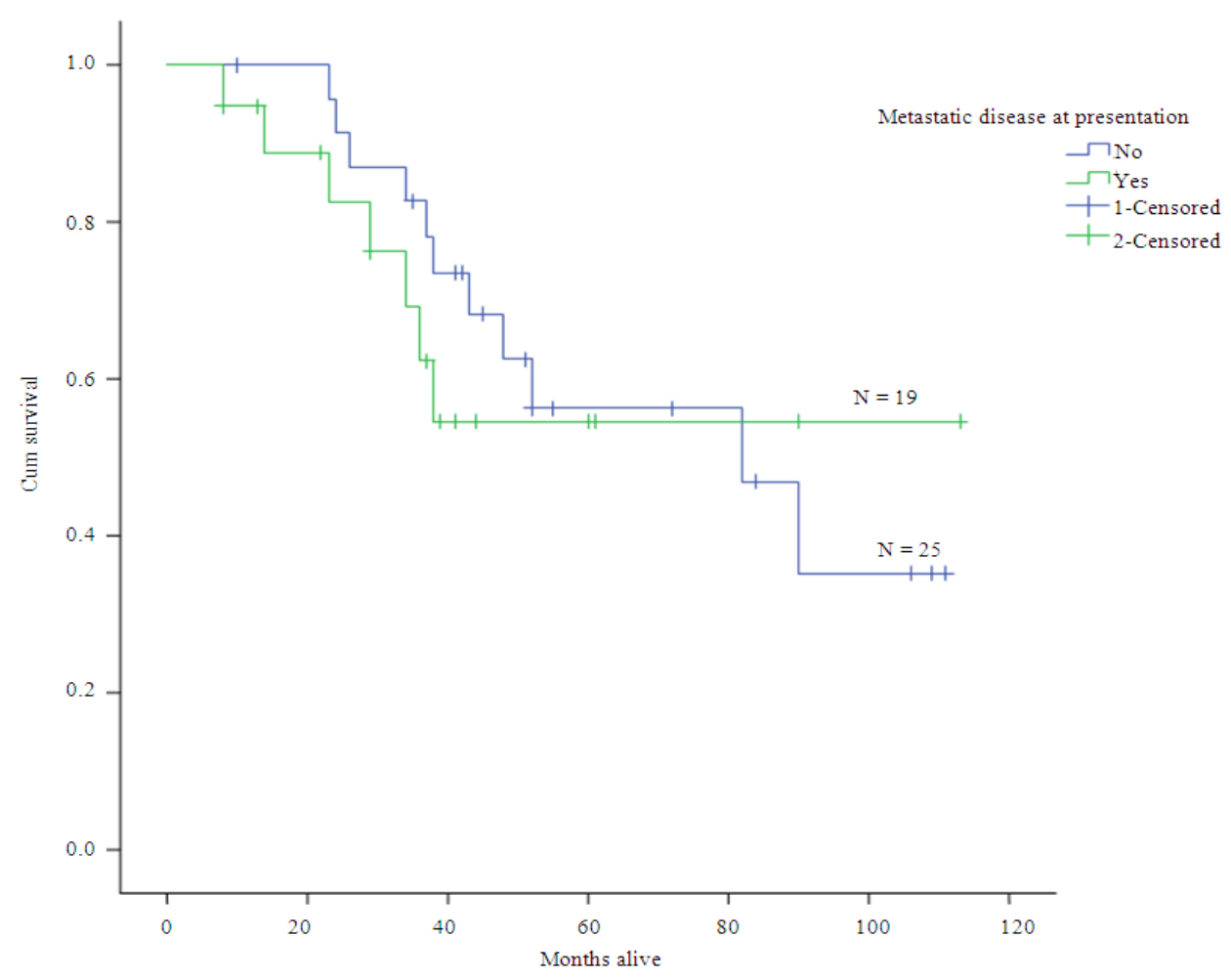

Fig. 3: Kaplan-Meier plot showing overall survival with respect to metastatic status at first presentation

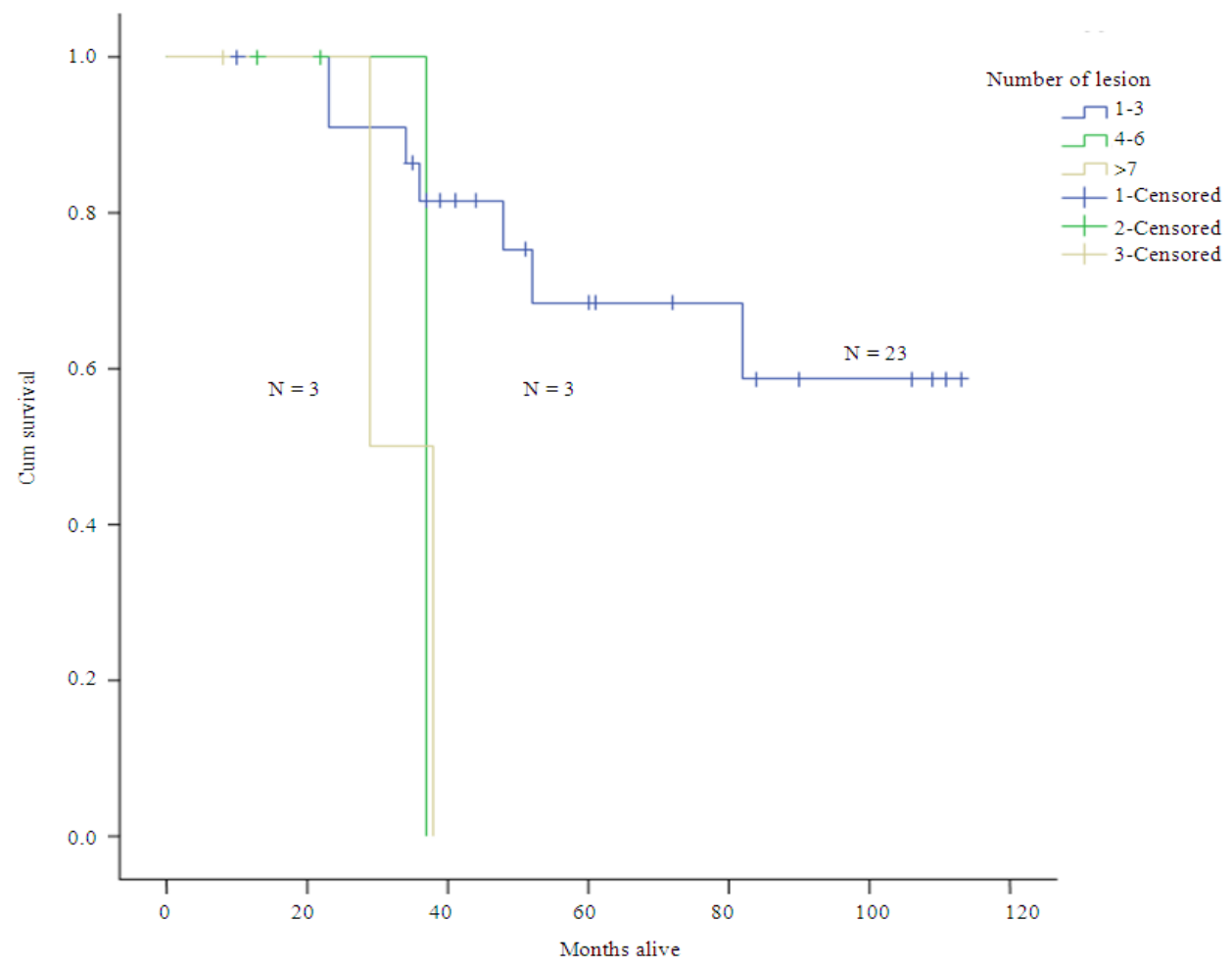

Fig. 4: Kaplan-Meier plot showing the survival with respect to the number of metastases at presentation 
Am. J. Immunol., 8 (1): 18-26, 2012

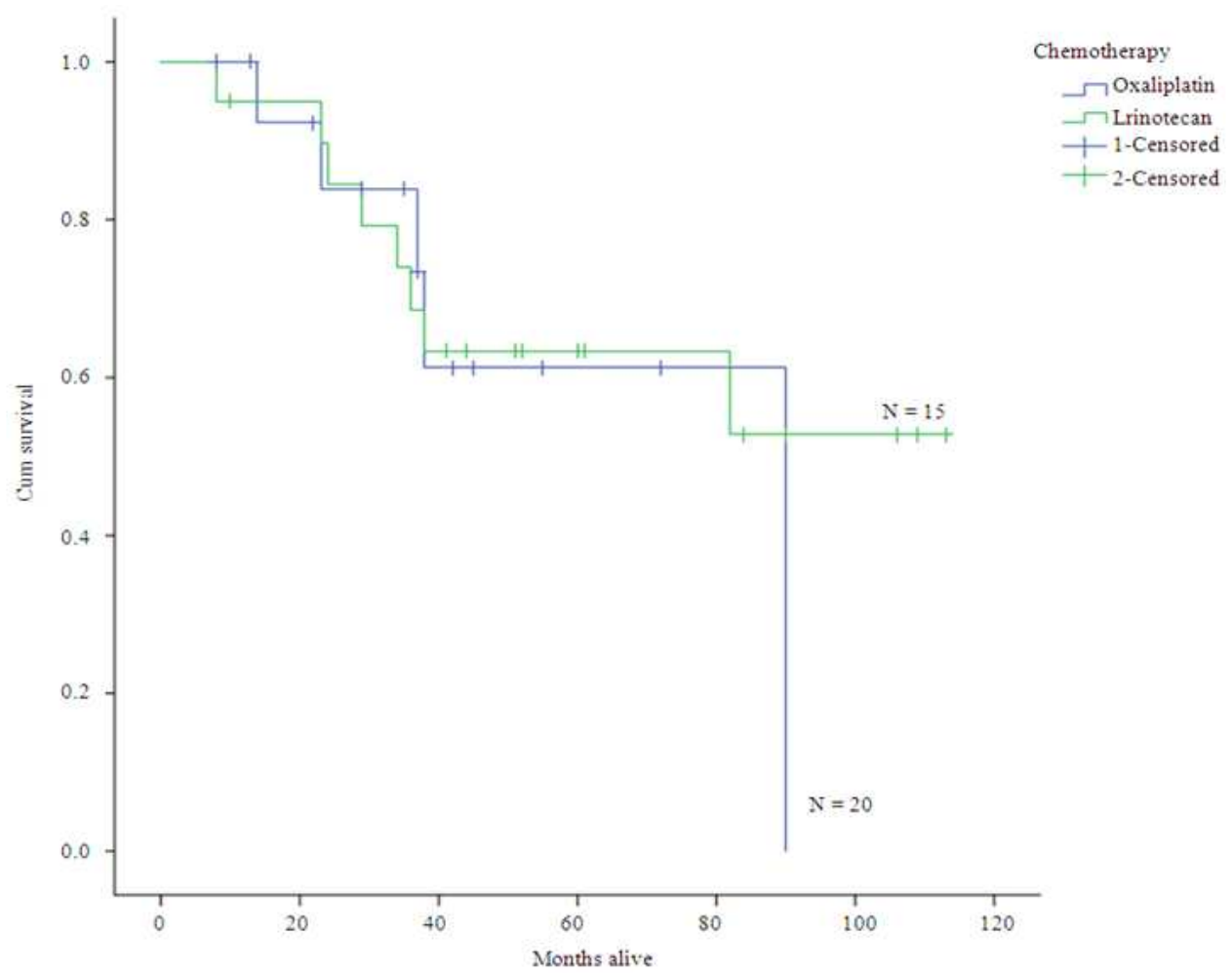

Fig. 5: Kaplan-Meier plot showing overall survival with respect to first line chemotherapy received

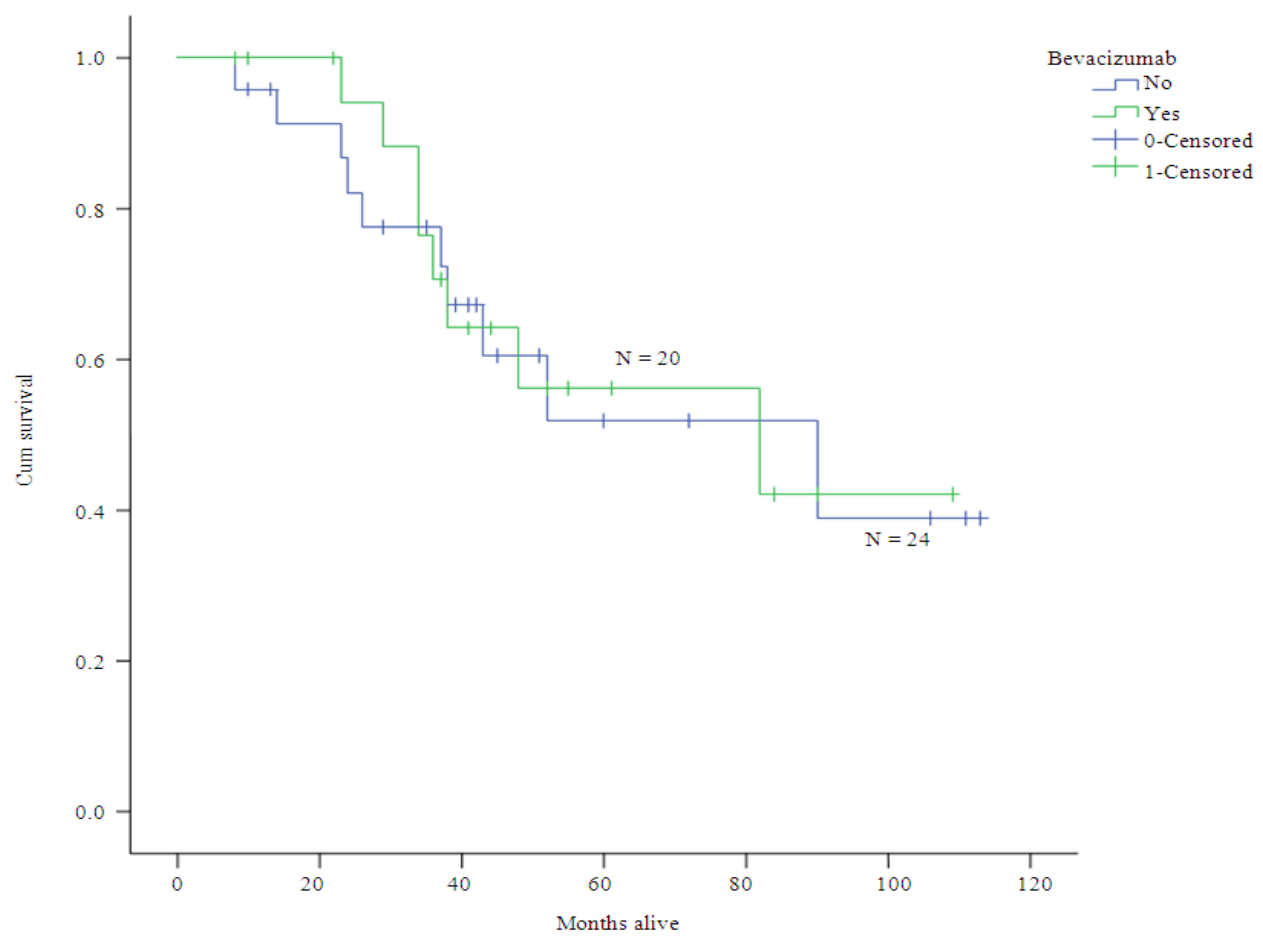

Fig. 6: Kaplan-Meier plot showing overall survival with respect to biological therapy 
Am. J. Immunol., 8 (1): 18-26, 2012

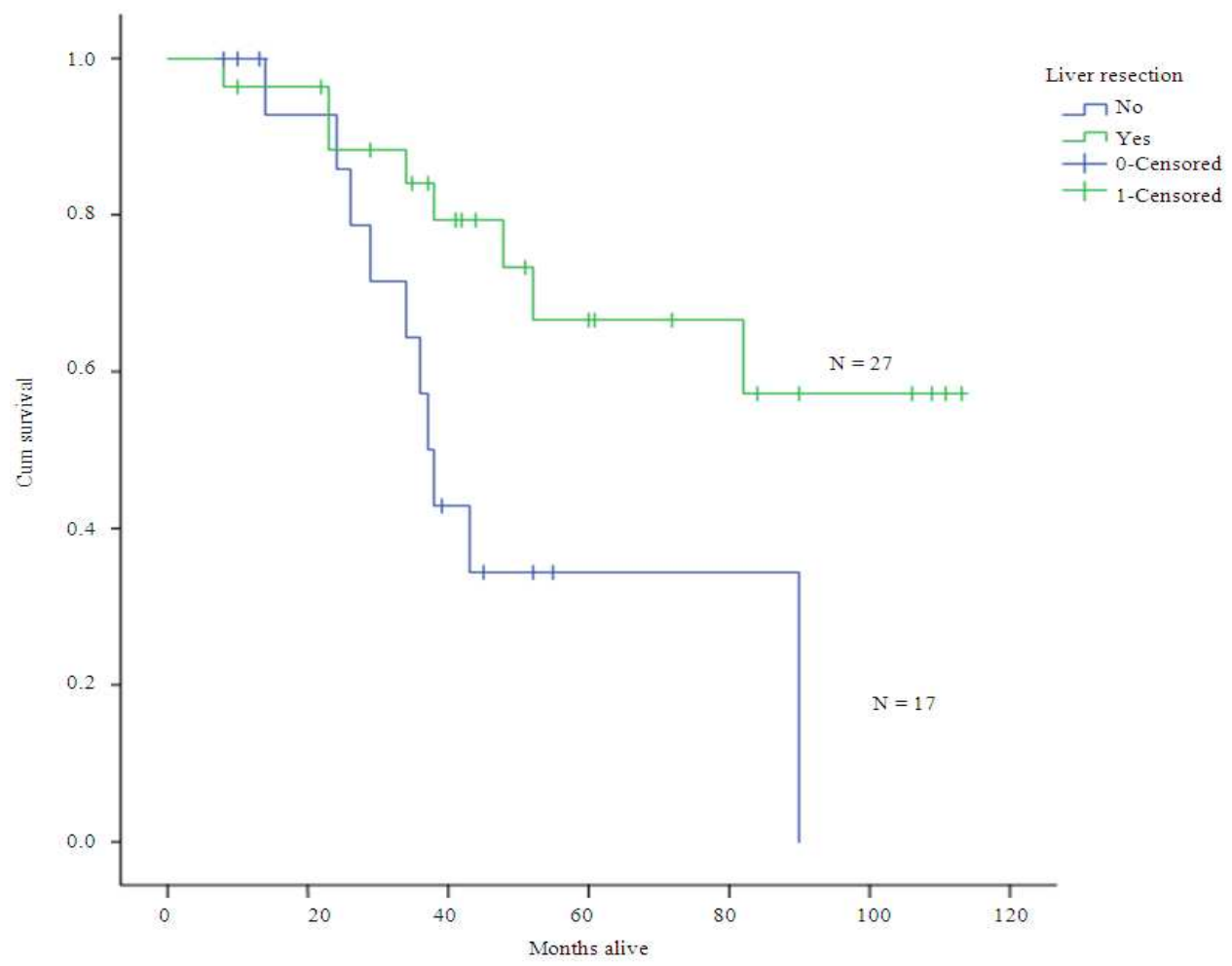

Fig.7: Kaplan-Meier plot showing overall survival with respect to surgical therapy

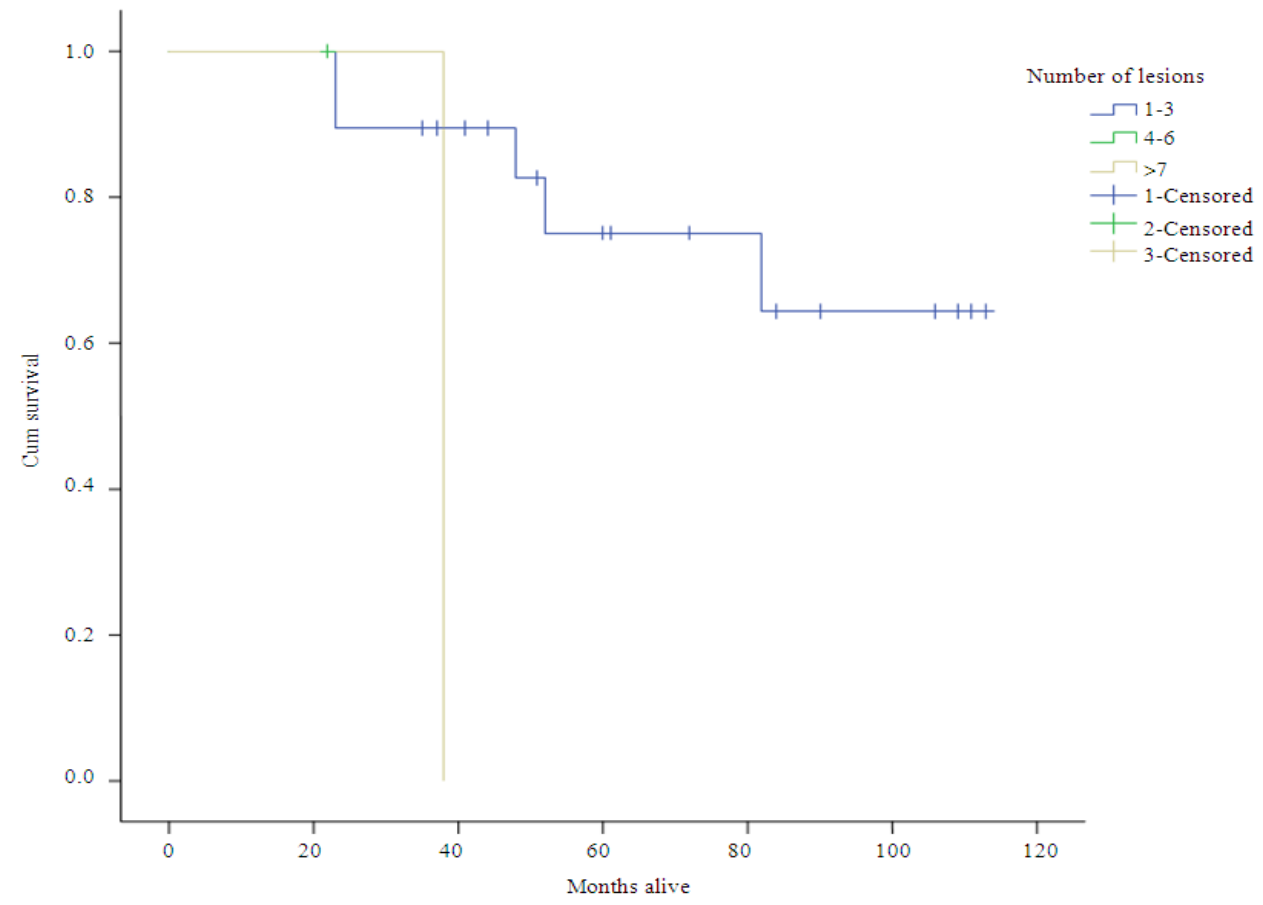

Fig. 8: Kaplan-Meier plot showing overall survival for patients who had undergone surgery with respect to the number of hepatic lesions 
Am. J. Immunol., 8 (1): 18-26, 2012

Table 2: Number of hepatic metastases for patients with hepatic-only MCRC

\begin{tabular}{lcc}
\hline Radiological Features & Number & Percentage \\
\hline$<3$ lesions & 23 & $52.30 \%$ \\
4-6 lesions & 3 & $6.80 \%$ \\
$>7$ lesions & 3 & $6.80 \%$ \\
Information not available & 15 & $34.10 \%$ \\
Total & 44 & $100.00 \%$ \\
\hline
\end{tabular}

Table 3: Therapeutic options for patients with hepatic-only MCRC

\begin{tabular}{lcc}
\hline Therapeutics options & Number & Percentage \\
\hline Chemotherapy & 15 & \\
First line therapy including Oxaliplatin & 20 & 34.10 \\
First line therapy including Irinotecan & 9 & 45.50 \\
First line therapy without Oxaliplatin or Irinotecan & & 20.50 \\
Biological therapy & 20 & 45.50 \\
With Bevacizumab & 24 & 54.50 \\
Without Bevacizumab & 27 & 61.40 \\
Surgical therapy & $17^{\text {a }}$ & 38.60 \\
Hepatic lesion resection performed & \\
Hepatic lesion resection not performed &
\end{tabular}

Statistical review for type of first line chemotherapy agent received showed no difference with respect to overall survival $(\mathrm{p}>0.05)$.

Statistical review for addition of biological therapy to chemotherapy as a therapeutic option showed no difference to overall survival ( $\mathrm{p}>0.05$ ).

Statistical review for use of hepatic metastases resection showed that patients receiving this modality had better overall survival ( $\mathrm{p}<0.001)$. The median overall survival for those who did not undergo hepatic metastases resection was 37.0 months, the mean overall survival for those who had undergone surgery was 84.1 months (mean used, as median not reached). Nonactuarial 5-year survival for patients undergoing hepatic surgery was $65 \%$.

Those patients who had successfully undergone hepatic surgery, were looked at separately and the survival based on the number of lesions noted on radiology revealed that those with fewer lesions had a better outcome and are displayed in Fig. 8.

Statistical review was undertaken, but did not confirm any survival advantage based on the number of hepatic lesions noted pre-operatively, but this might be related to the size of the sample being used.

The median follow-up for all patients involved was 40.0 months, with a range of 8.0 to 113.0 months.

\section{DISCUSSION}

Hepatic metastatic disease is a relatively common finding in patients with colorectal cancer, accounting for almost $30 \%$ of cases (Kopetz et al., 2009) but the proportion of these cases that are suitable or can be made suitable for hepatic resection in comparison is not as high, with some studies quoting between $10-25 \%$ resection rates (Barugel et al., 2009; Ksienki et al., 2010).
The main objective of this study was to review our own experience and to see how it compared to documented cases within the literature. Our results revealed that the mean age at presentation for hepatic-only MCRC was 60.8 years, with a male preponderance. This is comparable to findings in the literature (Al-Sahaf et al., 2009; Ozturk et al., 2010).

Degree of histological differentiation was found to be a significant predictor of overall survival, in keeping with the published data (Wang et al., 2010; Stillwell et al., 2011). In this patient series, T-stage of the primary CRC and the nodal status did not predict overall survival. This was contrary to published reports in which the T-stage and $\mathrm{N}$-stage have been identified as indicators of survival, this may be due to the small sample size (Stillwell et al., 2011; Wong et al., 2010). Error! Bookmark not defined. Moreso, current reports reflect that positive metastatic to examined Lymph Nodes Ratio (LNR) was an independent factor for overall survival in MCRC and is superior to the N-stage evaluation on its own (Wong et al., 2010).

The majority $(56.8 \%)$ of the patients within the group were detected on surveillance following adjuvant therapy. This is almost comparable to what can be found within peer-reviewed publications (Hayashi et al., 2010). Within the literature, hepatic metastases larger than $5 \mathrm{~cm}$, bilobar hepatic metastases or more than 3 metastases are considered to reflect a high tumour burden and are offer associated with a poorer prognosis (Ksienki et al., 2010; Wang et al., 2010) within our cohort, this feature of high tumour burden, relative to the number of metastases was reviewed and shows that patients with more than 3 hepatic metastases had a poorer overall survival $(\mathrm{P}>0.05)$.

Approximately $25 \%$ of the patients within the group underwent EGFR testing, with $45 \%$ having mutant-type. The uptake for this testing is rather low, because of change in standard practise for patients with MCRC, who might be suitable for biological therapy with such agents as cetuximab or more recently panitumumab. Based on reviews in the literature, EGFR mutations can be seen in $27-43 \%$ of some populations with MCRC (Siddiqui and Pipderdi, 2010).

Determining the best treatment pathway for patients with MCRC, is not a 'cut and dry' issue, with all patients fitting into the same mould and as such requires multidisciplinary discussion, to identify the best course and sequence of treatment (Cutsem et al., 2010). The introduction of new combination chemotherapy involving within the last decade has improved the outcomes and survival, but enabling 10-20\% with primarily unresectable hepatic-only metastases can be converted to become amenable for surgical intervention (Barugel et al., 2009; Tsoulfas et al., 2011) Within our group, these was a preponderance for patients to have 
Am. J. Immunol., 8 (1): 18-26, 2012

irinotecan-based chemotherapy $(45.5 \%)$ as apposed to oxaliplatin-based chemotherapy (34.1\%) or fluoropyrimidime monotherapy $(20.5 \%)$ and this appeared to have no impact on overall survival. However, from some sources such as Kim et al. (2009) patients who had oxaliplatin- based regimes after hepatic resection, had better DFS as compared to those on irinotecan-based regimes or fluoropyrimidime monotherapy (Kim et al., 2009) as our group was small, we did not review survival based on timing of chemotherapy before or after surgery, as the samples would have been small for statistical analysis.

The use of the anti-Vascular Endothelial Growth Factor (VEGF) antibody was employed and there was no statistical advantage obseved within our group. It has been noted the that this agent, increases the activity of a cytotoxic regimen and has been shown to improve overall survival, Progression-Free Survival (PFS) and response rate in the first-line setting for MCRC (Cutsem et al., 2010) Surgical resection with clear and negative margins remains the gold standard for treatment in hepatic-only CRC (Tonelii et al., 2010). Resection of hepatic metastases seemed to be the major factor for overall survival within our group, with those undergoing resection having a mean overall survival of almost 84.1 months, as compared to 37 months for those who did not. This compares to what is reported by Kopetz et al. (2009) where median overall survival was 65.3 months and 26.7 months respectively; this is tempered by the fact that there has been discordance in mean and median overall survival between population and instituational databases, which may be due to inherent limitations of single institutional results.3 The 5-year survival for patients undergoing surgical intervention as part of their CMT seems comparable with some reports in literature, but can vary from 9-63\% based on study (Cutsem et al., 2010; Simmonds et al., 2006)

This retropective review is limited by the number of patients identified within our unit at MWRH, but it offers good insight into our patient demographics and that mostly they compare favourably with published information within the literature. The next question to be addressed, once a larger cohort can be amassed, is the appropriate timing and sequencing of therapeutic options for patients with hepatic-only MCRC.

\section{CONCLUSION}

- Hepatic resection of metastases is the best therapeutic and can influence overall survival for patients with hepatic-only MCRC

- Chemotherapy remains a good adjunct to surgical intervention for patients with hepatic-only MCRC, but within our group it does not offer any additional survival advantage when not used in conjucntion with surgical therapy

\section{REFERENCES}

Al-Sahaf, O., D. Al-Azawi, A. Al-Khudairy, M.Z. Fauzi and S. El-Masry et al., 2009. Referral patterns of patients with liver metastases due to colorectal cancer for resection. Int. J. Colorectal Dis., 24: 79-82. DOI: 10.1007/s00384-008-0561-6

Barugel, M.E., C. Vargas and W.K. Waltier, 2009. Metastatic colorectal cancer: Recent advances in its clinical management. Expert Rev. Anticancer Ther., 9: 1829-1947. DOI: 10.1586/era.09.143

Cutsem, E.V., B. Nordlinger and A. Cervantes, 2010. Advanced colorectal cancer: ESMO Clinical Practice Guidelines for treatment. Ann. Oncol., 21: v93-v97. DOI: 10.1093/annonc/mdq222

Hayashi, M., I. Yoshihiro, K. Koji, T. Shimizu and M. Asakuma et al., 2010. Clinicopathological analysis of recurrence patterns and prognostic factors for survival after hepatectomy for colorectal liver metastasis. BMC Surgery, 10: 27-27. DOI: 10.1186/1471-2482-10-27

Kim, S.Y., H.J. Kim, Y.S. Hong, K.H. Jung and J.W. Park et al., 2009. Resected colorectal liver metastases: Does the survival differ according to postoperative chemotherapy regimen? J. Surg. Oncol., 100: 713-718. DOI: 10.1002/jso.21403

Kopetz, S., G.J. Chang, M.J. Overman, C. Eng and D.J. Sargent et al., 2009. Improved survival in metastatic colorectal cancer is associated with adoption of hepatic resection and improved chemotherapy. J. Clin. Oncol., 27: 3677-3683. DOI: $10.1200 / \mathrm{JCO} .2008 .20 .5278$

Ksienki, D., R. Woods, C. Speers and H. Kennecke, 2010. Patterns of referral and resection among patients with liver-only Metastatic Colorectal Cancer (MCRC). Ann. Surg. Oncol.,17: 30853093. DOI: 10.1245/s10434-010-1304-9

Ozturk, E., S. Kilicturgay, T. Yilmazlar, A. Zorluoglu and Y. Ozen, 2010. Factors affecting the prognosis of patients with colorectal liver metastasis. Acta Chir. Belg., 110: 189-194. PMID: 20514831

Power, D.G. and N.E. Kemeny, 2010. Role of adjuvant therapy after resection of colorectal cancer liver metastases. J. Clin. Oncol., 28: 2300-2309. DOI: 10.1200/JCO.2009.26.9340

Siddiqui, A.D. and B. Pipderdi, 2010. KRAS mutation in colon cancer: A marker of resistance to EGFR-I therapy. Ann. Surg. Oncol., 17: 1168-1176. DOI: 10.1245/s10434-009-0811-z 
Simmonds, P.C., J.N. Primrose, J.L. Colquitt, O.J. Garden and G.J. Poston et al., 2006. Surgical resection of hepatic metastases from colorectal cancer: A systematic review of published studies. Br. J. Cancer, 94: 982-999. PMID: 16538219

Stillwell, A.P., P.G. Buettner, S.K. Siu, R.W. Stitz and A.R.L. Stevenson et al., 2011. Predictors of postoperative mortality, morbidity, and long-term survival after palliative resection in patients with colorectal cancer. Dis. Colon Rectum, 54: 535-544. DOI: 10.1007/DCR.0b013e3182083d9d

Tonelii, F., F. Leo, S. Nobili, E. Mini and G. Batignani, 2010. Prognostic factors in primary and iterative surgery of colorectal liver metastases. J. Chemother., 22: 358-363. PMID: 21123161
Tsoulfas G., M.G. Pramateftakis and I. Kanellelos, 2011. Surgical treatment of hepatic metastases from colorectal cancer. World J. Gastrointest Oncol., 3: 1-9. DOI: 10.4251/wjgo.v3.i1.1

Wang, Y., Y.F. Liu, Y. Cheng, D.H. Yi and P. Li et al., 2010. Prognosis of colorectal cancer with liver metastasis: Value of a prognostic index. Braz. J. Med. Biol. Res., 43: 1116-1122. DOI: 10.1590/S0100-879X2010007500103

Wong, K.P., J.T.C. Poon, J.K.M. Fan and W.L. Law, 2010. Prognostic value of lymph node ratio in stage III colorectal cancer. Colorectal Dis., 13: 11161122. DOI: $10.1111 /$ j.1463-1318.2010.02435.x 